\title{
NanoESI Mass Spectrometry of Rubisco and Rubisco Activase Structures and Their Interactions with Nucleotides and Sugar Phosphates
}

\author{
Michelle J. Blayney, ${ }^{1}$ Spencer M. Whitney, ${ }^{2}$ Jennifer L. Beck ${ }^{1}$ \\ ${ }^{1}$ School of Chemistry, University of Wollongong, New South Wales 2522, Australia \\ ${ }^{2}$ Plant Science Division, Research School of Biology, Australian National University, Canberra, Australia
}

\begin{abstract}
Ribulose bisphosphate carboxylase/oxygenase (Rubisco) is the protein that is responsible for the fixation of carbon dioxide in photosynthesis. Inhibitory sugar phosphate molecules, which can include its substrate ribulose-1,5-bisphosphate (RuBP), can bind to Rubisco catalytic sites and inhibit catalysis. These are removed by interaction with Rubisco activase (RA) via an ATP hydrolytic reaction. Here we show the first nanoESI mass spectra of the hexadecameric Rubisco and of RA from a higher plant (tobacco). The spectra of recombinant, purified RA revealed polydispersity in its oligomeric forms (up to hexamer) and that ADP was bound. ADP was removed by dialysis against a high ionic strength solution and nucleotide binding experiments showed that ADP bound more tightly to RA than AMP-PNP (a non-hydrolysable ATP analog). There was evidence that there may be two nucleotide binding sites per RA monomer. The oligomerization capacity of mutant and wild-type tobacco RA up to hexamers is analogous to the subunit stoichiometry for other AAA+ enzymes. This suggests assembly of RA into hexamers is likely the most active conformation for removing inhibitory sugar phosphate molecules from Rubisco to enable its catalytic competency. Stoichiometric binding of RuBP or carboxyarabinitol bisphosphate (CABP) to each of the eight catalytic sites of Rubisco was observed.
\end{abstract}

Key words: Rubisco, Rubisco activase, Molecular chaperone, ATPase, AAA+ ATPase, Noncovalent complex, Protein-protein interaction, Electrospray

\section{Introduction}

$\mathrm{R}$ ibulose bisphosphate carboxylase/oxygenase (Rubisco) is the enzyme responsible for catalysis of the first step of carbon dioxide assimilation in the Calvin cycle of photosynthesis [1-3]. In this cycle, ATP and NADPH from

Electronic supplementary material The online version of this article (doi:10.1007/s13361-011-0187-8) contains supplementary material, which is available to authorized users.

Correspondence to: Jennifer L. Beck; e-mail: jbeck@uow.edu.au the light reactions of photosynthesis are used for the reduction of $\mathrm{CO}_{2}$ to produce carbohydrates. In the first phase, carbon dioxide within the chloroplast is fixed to ribulose bisphosphate (RuBP), then cleaved to form two molecules of 3-phosphoglycerate. This multistep reaction is catalysed by Rubisco [2, 4]. In the second phase, 3phosphoglycerate is phosphorylated by ATP and the resulting product is reduced by NADPH to yield glyceraldehyde3-phosphate, which is used to produce carbohydrates. In the final phase of the cycle, RuBP is regenerated in a series of reactions catalysed by various enzymes to allow the cycle to continue. In most plants, flux through this cycle is often 
limited by the relatively poor catalytic performance of Rubisco that necessitates plants to produce substantial quantities of it, investing $20 \%$ to $50 \%$ of their leaf protein into Rubisco [1,5]. As a result of this investment, Rubisco is the most abundant protein on Earth [6] and a valued target for catalytic improvement in crops to increase yield [7].

Rubiscos of higher plants and cyanobacteria form hexadecameric quaternary structures, consisting of eight large subunits $(51-58 \mathrm{kDa})$ and eight small subunits (12$18 \mathrm{kDa}$ ) [5]. They are arranged as four large subunit dimers capped at each end with four small subunits [8]. The catalytic site is located between the C-terminal domain of one large subunit and the N-terminal region of the other large subunit in the dimer [8]. Rubisco requires activation by a $\mathrm{CO}_{2}$ molecule in addition to the substrate $\mathrm{CO}_{2}$ [9]. The activating $\mathrm{CO}_{2}$ reacts with a conserved lysine residue in the catalytic site forming a carbamate, which is involved with other residues and water molecules to coordinate $\mathrm{a} \mathrm{Mg}^{2+}$ ion [4]. X-ray crystal structures show that the binding of RuBP to the activated catalytic site is accompanied by movement of a mobile structural element (loop-6) that "closes" over the active site to facilitate the catalytic chemistry of Rubisco [4]. $\mathrm{RuBP}$ is also able to bind tightly but unproductively to the noncarbamylated (inactive) form of Rubisco making RuBP both a substrate and an inhibitor of the enzyme [10]. Binding of RuBP to inactive Rubisco stalls catalysis and prevents activation via carbamylation and $\mathrm{Mg}^{2+}$ binding [10]. Inhibitory RuBP is removed from the catalytic site by the action of the enzyme, Rubisco activase [11].

Rubisco activase (RA) regulates Rubisco activity by facilitating the dissociation of RuBP and other inhibitory sugar phosphates from Rubisco catalytic sites to reinstall its catalytic capacity [11-13]. The three-dimensional structure of RA has not yet been determined and the mechanism of activation of Rubisco by RA is as yet unknown. Nevertheless, some of the biochemical properties of RA and sequence similarities to other proteins point to possible modes of action. RA exhibits ATPase activity [14, 15], and has been identified as an AAA+ protein [16]. AAA+ proteins (ATPases associated with a variety of cellular activities) are a class of ATPases associated with the assembly, operation and disassembly of protein complexes [16]. AAA+ proteins generally form ring-shaped oligomers (usually hexameric) and this arrangement is important for their ATPase activities and modes of action [16-18]. Importantly, the ATPase activity of RA is not dependent on Rubisco but is regulated by physiological ADP/ATP ratios in chloroplasts and, in some plants, via light dependent redox regulation of some RA isoforms [11, 19, 20].

Several studies indicate that RA activity is enhanced by oligomeric assembly. Early gel filtration experiments demonstrated self-association of RA in the presence of polyethylene glycol (PEG) [21]. In the same work, the Rubisco activation capacity and ATPase activities of RA were shown to be enhanced with increasing enzyme concentrations, which implied that self-association stimulated RA function- ality. Similarly, changes in intrinsic fluorescence (Trp residues) and stimulation of ATPase activity consistent with aggregation of RA have been observed [22, 23]. Experiments carried out to confirm the size or preferred oligomeric form of RA have produced varied results, dependent on the conditions or method used. Gel filtration chromatography showed that increased aggregation of spinach RA occurred in the presence of ATP and excess $\mathrm{Mg}^{2+}$ [22]. Characterisation of the size of the assembly was difficult as the protein eluted as an asymmetric peak, indicating a mixture of oligomeric forms (proposed to constitute 8-14 RA monomers) was present, depending on the temperature and availability of sugar phosphate ligands.

The activation of Rubisco by RA is therefore complex and there are many factors to be considered to arrive at a molecular mechanism. The activation requires hydrolysis of ATP by RA with activation being inhibited by ADP. The ATPase activity requires binding of ATP and $\mathrm{Mg}^{2+}$ by RA which is thought to be accompanied by a conformational change and aggregation to a higher order oligomeric form, leading to the hydrolysis of ATP [24 and references therein]. The activation requires some kind of interaction between the two proteins $[9,23]$. It is proposed that the conformational change of RA associated with ATP binding/hydrolysis stimulates a conformational change in Rubisco [18]. Several studies have supported a physical association between Rubisco and RA. For example, RA shows specificity for particular Rubiscos such that RA from Solanaceae species (e.g., tomato, eggplant, capsicum, chili, etc.) cannot efficiently activate Rubisco from a non-Solanaceae species [12]. Comparisons of the Rubisco large subunit sequences from Solanaceae and from non-Solanaceae species showed amino acid differences in their $\mathrm{N}$-domain that reside on the holoenzyme's surface close to the catalytic sites [25]. Mutagenesis studies showed that a single residue (Pro 89) located on the N-terminus of Solanaceae Rubisco large subunits was required for activation by RA [26]. Chemical cross-linking has also been used to show that RA can bind to the large subunit of Rubisco [27], and co-immunoprecipitation of the two proteins has been demonstrated [28]. Studies involving a chimeric RA (with spliced regions from tobacco and spinach RA) indicate that the RA C-terminal domain was the main determinant of Rubisco species specificity [29]. These results confirm that at some point during the process of Rubisco activation, a Rubisco-RA complex is present. Owing to the transient nature of this relationship, detail on the stoichiometry and mechanism of the interaction has yet to be elucidated.

Several models for the RA activation mechanism have been put forward $[9,13,30]$. These models do not explain exactly how RA facilitates the release of inhibitors from Rubisco. It is unclear whether RA actively binds the inhibitory sugar phosphates directly or whether RA causes a conformational change to Rubisco that stimulates their dissociation. The mechanism by which RA complexed with ADP can bind ATP is also ambiguous. This may also occur 
via a conformational change associated with ATP binding that decreases the affinity of RA for ADP facilitating its release. These questions need to be addressed in order to conclusively determine the RA mechanism.

There are conflicting reports in the literature on the effects of ATP/ADP on the oligomerization of RA and its activation of Rubisco. While the addition of ATP and $\mathrm{Mg}^{2+}$ increased the intrinsic fluorescence (proposed to correspond to the extent of oligomerization) of spinach RA, a significantly smaller increase was observed when either ATP or $\mathrm{Mg}^{2+}$ alone was used [22]. In order to investigate the effect of nucleotide binding on tobacco RA, arginine residues (R241, R244, R294, R296) proposed to be located in the ATP-binding domain were mutated to alanine, which reduced, or eliminated, ATPase activity [24]. The apparent molecular masses of wild type tobacco RA (and the arginine mutants) were similar in the presence of ATP or ADP suggesting that nucleotide binding had little or no effect on oligomerization. In contrast, gel filtration analysis indicated ATP stimulated oligomerization of spinach RA and an ATPase deficient R244A mutant analog, although no conformational change was apparent by changes in intrinsic fluorescence [24]. These contrasting results suggest the ATP-induced increase in intrinsic fluorescence of tobacco RA may correspond to reorientations between subunits within an oligomeric complex rather than assembly of the subunits into larger oligomers. It was also suggested that larger oligomers may form transiently during ATP binding/ hydrolysis which may not be detected by these methods [24].

The methods that have previously been used to investigate nucleotide binding and the extent of RA oligomerization yielded average stoichiometries for these processes. Electrospray ionization mass spectrometry (ESI-MS) has advanced to a stage where large oligomeric assemblies and noncovalent protein-ligand complexes can be studied and stoichiometries determined [31]. There have been several studies in which ESI-MS has been applied to AAA+ proteins to examine oligomerization and interactions with ligands such as nucleotides and DNA. Previously we reported the effect of mutating key amino acid residues on the order of oligomerization (hexamer, heptamer, or higher order oligomers) of the Escherichia coli DnaB helicase protein [32]. Complexes of DnaB with DnaC, the protein used as a binding partner for loading DnaB onto DNA were observed (e.g., $\left.\mathrm{DnaB}_{6}\left(\mathrm{DnaC}_{6}\right)\right)$, in addition to complexes of $\mathrm{DnaB}_{6}$ with up to six molecules of ADP bound to the hexamer (from samples where DnaB was prepared with ATP) consistent with the previously described ATPase activity of DnaB. Batchelor et al. showed that a $\sigma(54)$ activator protein that forms an active ATPase ring assembly to enable transcription by a $\sigma(54)$ RNA polymerase formed a hexamer while its isolated ATPase domain formed heptamers [33]. Such studies provide insight into the mechanisms by which these proteins "count." The counting mechanism is important to their function since the correct assembly of these protein rings assists the correct assembly and function of their targeted protein binding partners.

In the current work, conditions are described for the nanoelectrospray ionization mass spectrometry (nanoESIMS) analysis of tobacco Rubisco and RA. Here we examine by nanoESI-MS the tight binding of ADP to purified recombinant RA and the possible implications this unaccounted binding might have on previous work examining RA-nucleotide binding. A method is described for removal of ADP from RA that enabled comparison by nanoESI-MS of the relative binding affinities of ADP, adenosine-5'-( $\beta, \gamma$-imido)-triphosphate (AMP-PNP), adenosine-5'-( $\gamma$-thio)-triphosphate (ATP $\gamma$ S) and adenosine-5'triphosphate (magnesium salt, ATP-Mg) and their influence on oligomerization. Also examined are the relative binding affinities and oligomerization effects of these nucleotides on RA containing single (R241A, R296A, $\mathrm{R} 294 \mathrm{~A})$ and triple $(\mathrm{R}[241,244,296] \mathrm{A})$ mutations at residues thought to be involved in nucleotide binding. Subsequent analysis of active and RuBP- or carboxyarabinitol bisphosphate (CABP)-inhibited Rubisco by nanoESI-MS both before and after incubation with RA is used to confirm its hexadecameric structure and examine the binding stoichiometries of both ligands and the capacity of RA to remove RuBP. These studies highlight the utility of ESI-MS for providing new insights into the interactions of large protein assemblies.

\section{Experimental}

\section{Materials}

MilliQ water from Millipore (Molsheim, France) was used in all experiments. Magnesium acetate $\left[\mathrm{Mg}(\mathrm{OAc})_{2}\right]$, ethylenediaminetetraacetic acid (free acid, EDTA), guanidine hydrochloride, adenosine 5'-triphospho(enol)pyruvic acid (monopotassium salt, PEP), sodium hydrogen carbonate $\left(\mathrm{NaHCO}_{3}\right)$, creatine phosphate (dibasic tetrahydrate), adenosine triphosphate (magnesium salt, ATP-Mg), adenosine 5'diphosphate (free acid, ADP), adenosine 5 '-( $\beta, \gamma$-imido) triphosphate (free acid, AMP-PNP) and adenosine-5'-( $\gamma$ thio)-triphosphate (free acid, ATP $\gamma$ S), were purchased from Sigma-Aldrich (St. Louis, MO, USA). Ammonia, ammonium acetate $\left(\mathrm{NH}_{4} \mathrm{OAc}\right)$, acetic acid, formic acid, and methanol (HPLC grade) were obtained from Ajax Finechem (Seven Hills, Australia). Tris(hydroxymethyl)aminomethane (Tris) was obtained from ICN Biomedicals (now MP Biomedicals; Aurora, OH, USA). Nicotinamide adenine dinucleotide (NADH), pyruvate kinase (PK), and lactate dehydrogenase (LDH) were purchased from Roche Diagnostics (Castle Hill, Australia). Dialysis tubing [10,000 molecular weight cut off (MWCO)] was purchased from Crown Scientific (Moorebank, Australia). Slide-A-Lyzer dialysis cassettes $(10,000$ MWCO, $0.1-0.5 \mathrm{~mL})$ and SlideA-Lyzer mini dialysis units (7000 MWCO, 10-100 $\mu \mathrm{L})$ were purchased from Thermo Scientific (IL, USA). Milli- 
pore Biomax centrifugal filters (5000 MWCO) were obtained from Millipore.

Preparation and Purification of RuBP, CABP, Rubisco, Rubisco Activase, and Rubisco Activase Mutants Ribulose1,5-bisphosphate (RuBP) was synthesized from ribose-5phosphate and purified as described [34]. The tight binding Rubisco inhibitor 2-carboxyarabinitol 1,5-bisphosphate (CABP) was synthesized and purified as described [35]. Rubisco isolated from Nicotiana tabacum (tobacco) leaves was purified as described [36] and stored at $-80{ }^{\circ} \mathrm{C}$ in $40 \mathrm{mM}$ Tris-HCl buffer, $\mathrm{pH} 8$ and 20\% (vol/vol) glycerol. The concentrations of Rubisco and Rubisco activase were determined using a Bio-Rad DC protein assay against bovine serum albumin standards. ATPase activity was measured with a spectrophotometric assay as described [14] where the production of ADP is measured through an enzyme-linked assay coupled to NADH oxidation. Prior to mass spectrometry, Rubisco samples ( $300 \mu \mathrm{L} ; 23 \mathrm{mg} / \mathrm{mL})$ were dialysed at $4{ }^{\circ} \mathrm{C}$ against three changes of $2 \mathrm{~L}$ of $100 \mathrm{mM}$ ammonium acetate solution, $\mathrm{pH}$ 7.2.

The expression plasmid pHueAct was used to express tobacco Rubisco activase (RA) as an N-terminal 6-histidineubiquitin $\left(\mathrm{H}_{6} \mathrm{Ub}\right)$ tagged protein in BL21(DE3) cells [37]. Pristine RA was purified by Ni NTA agarose chromatography (Qiagen) selection of $\mathrm{H}_{6} \mathrm{Ub}-\mathrm{RA}$ and the $\mathrm{H}_{6} \mathrm{Ub}$ sequence cleaved and then removed by a second Ni NTA agarose chromatography step as described previously [37]. The purified RA was stored at $-80{ }^{\circ} \mathrm{C}$ in $25 \mathrm{mM}$ Tris- $\mathrm{HCl}$ buffer, $\mathrm{pH} 8$ and 20\% (vol/vol) glycerol. Mutant tobacco RA enzymes were produced via mutagenesis of its gene $(r c a)$ in pHueAct using the QuikChange Site-Directed Mutagenesis Kit (Stratagene) and appropriate primers. All plasmids were sequenced using BigDye terminator sequencing at the Biomolecular Resource Facility, the Australian National University. Before ESI mass spectrometry, pure RA $(300 \mu \mathrm{L} ; \sim 60 \mu \mathrm{M})$ was dialysed at $4{ }^{\circ} \mathrm{C}$ against three changes of $2 \mathrm{~L}$ of $10 \mathrm{mM}$ ammonium acetate solution, $\mathrm{pH}$ 7.2. Aliquots of $\mathrm{NH}_{4} \mathrm{OAc}(5 \mathrm{M})$ and water were added to the RA samples to give final concentrations of $\mathrm{NH}_{4} \mathrm{OAc}$ of $100-1000 \mathrm{mM}$ as designated in the relevant sections of the text prior to MS analysis.

The integrity of the secondary structure of all proteins used in this work was assessed by circular dichroism (CD) spectroscopy. CD spectra were acquired using a Jasco J-810 spectropolarimeter. All spectra were recorded using a $0.1 \mathrm{~cm}$ quartz cell and the following parameters: $\lambda=250-190 \mathrm{~nm}$; recording speed $100 \mathrm{~nm} / \mathrm{min}$; response $4 \mathrm{~s}$; spectral band width $1 \mathrm{~nm}$; number of accumulated scans $=4$. All $C D$ spectra presented here were obtained after subtraction of the background. In all measurements, the temperature was kept at $20{ }^{\circ} \mathrm{C}$ using a Ratek thermoregulator water bath. The final concentration of Rubisco activase and Rubisco (dialysed under different conditions) was $\sim 5 \mu \mathrm{M}$ for all $\mathrm{CD}$ measurements.
Removal of ADP from $R A$ and Titration with Nucleotides Samples of RA $(\sim 60 \mu \mathrm{M})$ were dialysed at $4{ }^{\circ} \mathrm{C}$ against three changes of $250 \mathrm{~mL}$ of $40 \mathrm{mM}$ Tris-HCl buffer, $4 \mathrm{M}$ in $\mathrm{NaCl}, \mathrm{pH} \mathrm{7.2,} \mathrm{followed} \mathrm{by} \mathrm{two} \mathrm{changes} \mathrm{of} 2 \mathrm{~L}$ of $100 \mathrm{mM}$ ammonium acetate solution, $\mathrm{pH}$ 7.2. The resulting ADP-free samples of RA were titrated with increasing amounts of either ADP or AMP-PNP ( $1 \mathrm{mM}$

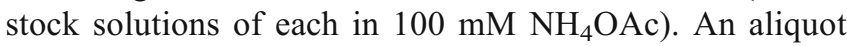
of $5 \mathrm{M}$ ammonium acetate, $\mathrm{pH} 7.2$, was added so that the final ammonium acetate concentration was $500 \mathrm{mM}$. Appropriate volumes of nucleotides (ADP and AMPPNP) were added to give final concentrations of 1,10 , and $100 \mu \mathrm{M}$. The concentration of ADP-free RA in the samples was approximately $30 \mu \mathrm{M}$ (if monomeric). Competitive nucleotide binding experiments were performed in three different ways. First, an aliquot of ADP was added to ADP-free RA and allowed to equilibrate for $10 \mathrm{~min}$ followed by AMP-PNP and equilibration for a further $10 \mathrm{~min}$ before a nanoESI mass spectrum was acquired. Second, an aliquot of AMP-PNP was added to ADP-free RA followed by ADP. Finally, the ADP-free RA was added to a mixture of ADP and AMP-PNP (so they were added simultaneously). The final concentrations of RA (if monomeric), nucleotide and $\mathrm{NH}_{4} \mathrm{OAc}$ in all samples were $30,100 \mu \mathrm{M}$, and $500 \mathrm{mM}$, respectively.

Preparation of $\mathrm{CO}_{2}$-Free (Noncarbamylated) Rubisco and Rubisco-RuBP Complex Prior to and during dialysis the buffers were sparged with $\mathrm{N}_{2}$ gas to ensure complete decarbamylation ("deactivation") of Rubisco. An aliquot of Rubisco $(23 \mathrm{mg} / \mathrm{mL})$ was dialysed at $4{ }^{\circ} \mathrm{C}$ against three changes of $400 \mathrm{~mL}$ of $100 \mathrm{mM}$ ammonium acetate solution, $1 \mathrm{mM}$ in EDTA, $\mathrm{pH} \mathrm{7.2,} \mathrm{then} \mathrm{four} \mathrm{changes} \mathrm{of} 400 \mathrm{~mL}$ of $100 \mathrm{mM}$ ammonium acetate solution, $\mathrm{pH}$ 7.2. To prepare samples of activity deficient Rubisco-RuBP complexes, $5 \mu \mathrm{L}$ of RuBP $(24.6 \mathrm{mM}$ in $3 \mathrm{mM} \mathrm{HCl})$ was added to a glass vial and freeze-dried before adding $100 \mu \mathrm{L}$ of $100 \mathrm{mM}$ ammonium acetate solution. After freeze drying again the vial was sealed with a septum seal cap and equilibrated with $\mathrm{N}_{2}$ gas before dissolving the RuBP in $50 \mu \mathrm{L} \mathrm{N}_{2}$-sparged $100 \mathrm{mM}$ ammonium acetate buffer. Aliquots of decarbamylated Rubisco were injected directly into the septum sealed vial containing RuBP and left to react on ice for $30 \mathrm{~min}$.

Attempts to Identify a Rubisco-RA Complex Attempts to prepare complexes of Rubisco with RA that could be distinguished by nanoESI-MS were carried out in several ways. Reactions containing $8 \mu \mathrm{M}$ of RA (with endogenous ADP bound, see below) was mixed with $200 \mu \mathrm{M}$ RuBP and $5 \mu \mathrm{M}$ Rubisco or $5 \mu \mathrm{M}$ Rubisco-RuBP complexes (prepared as described above) in $100 \mathrm{mM}$ ammonium acetate. Replica reactions were also prepared that contained $100 \mu \mathrm{M}$ ATP$\mathrm{Mg}$, AMP-PNP or ATP $\gamma$ S. Identical reactions containing $8 \mu \mathrm{M}$ of ADP-free RA (prepared as described above) instead of RA with endogenous ADP bound were also examined. 
NanoESI-MS Conditions Positive ion nanoESI mass spectra of native Rubisco activase, Rubisco activase with nucleotides, and Rubisco subunits were acquired using a Waters (Manchester, UK) extended mass range $(\mathrm{m} / \mathrm{z} 32,000)$ QTOF Ultima mass spectrometer, fitted with a Z-spray ESI source. The capillary and cone voltages were $1.5 \mathrm{kV}$ and $300 \mathrm{~V}$, respectively. The RF lens 1 and collision cell were $160-200 \mathrm{~V}$ and $4 \mathrm{~V}$, respectively, and the pressure in the ion optics region was 0.2 mbar. Positive ion nanoESI mass spectra of native Rubisco, Rubisco-RuBP, and RubiscoRuBP with RA (with or without nucleotides) were acquired using a Waters (Manchester, UK) Synapt HDMS mass spectrometer. Spectra were acquired with a capillary and cone voltage of $1.5 \mathrm{kV}$ and $200 \mathrm{~V}$, respectively. The trap collision energy and transfer collision energy were 6 and $4 \mathrm{~V}$, respectively, and the backing pressure was 0.36 mbar. Typically, 20-30 acquisitions were summed to obtain representative data. The data were calibrated using a solution of $10 \mathrm{mg} / \mathrm{mL}$ cesium iodide in $70 \%$ isopropanol.

\section{Results and Discussion}

\section{Molecular Mass of Rubisco Activase (RA)}

The molecular mass of the tobacco RA monomer (dialysed against $10 \mathrm{mM} \mathrm{NH} \mathrm{NH}_{4} \mathrm{OAc}$ ) was determined by addition of acetic acid to $13 \%$ (vol/vol) to disrupt the quaternary structure of the protein assembly prior to nanoESI-MS analysis. The resulting spectrum (Figure 1a) showed several charge state distributions suggesting a combination of partially folded protein structures present in the mixture, with the most abundant ions of the different folded forms corresponding to the $[\mathrm{RA}+24 \mathrm{H}]^{24+},[\mathrm{RA}+18 \mathrm{H}]^{18+}$, and $[\mathrm{RA}$ $+13 \mathrm{H}]^{13+}$ ions at $m / z 1783,2377$, and 3290 , respectively. The molecular mass of monomeric RA obtained from this mass spectrum was $42,760.1 \pm 0.3 \mathrm{Da}$ which matches the average molecular mass calculated from the sequence of tobacco RA $(42,759.6 \mathrm{Da})$ and attests to the sequence integrity of the recombinant RA purified using the $\mathrm{N}$ terminal 6xHis-tagged ubiquitin purification system [37].

Conditions were determined for nanoESI-MS of RA at $\mathrm{pH} 7.2$ in order to preserve the non-covalent interactions that maintain quaternary structure. The spectrum of RA $(\sim 30 \mu \mathrm{M}$ if monomeric) dialysed against $10 \mathrm{mM} \mathrm{NH}_{4} \mathrm{OAc}$ and adjusted to $500 \mathrm{mM} \mathrm{NH}_{4} \mathrm{OAc}$ just prior to analysis is shown in Figure 1b. This spectrum is very different from that obtained for RA in the presence of acid (Figure 1a), and from the normal charge state distribution commonly observed in ESI mass spectra for monomeric proteins or for monodisperse oligomeric proteins. The most likely oligomeric forms are indicated by numbers above the ions. It should be noted that assignment of oligomeric forms is complicated since, for example, the $10+$ ion for the dimer $\left([2 \mathrm{RA}+10 \mathrm{H}]^{10+}\right)$ occurs at the same value of $\mathrm{m} / \mathrm{z}$ as the $20+$ ion of the tetramer $\left([4 \mathrm{RA}+20 \mathrm{H}]^{20+}\right)$. The oligomeric order assigned in Figure 1b was also supported by the number of
ADP molecules bound to each form (see below). The presence of a range of oligomeric RA forms correlates with previous observations of the polydispersity in solution as judged by asymmetric elution of RA during size exclusion chromatography, and with a previous proposal that a dynamic equilibrium exists among RA monomers and oligomers under appropriate reaction conditions [23 and references therein]. When nanoESI mass spectra of RA were acquired at lower protein concentrations the higher oligomeric forms were less abundant (not shown). It cannot be ruled out that the multiple RA oligomers identified by ESIMS arise from dissociation of RA complexes in the mass spectrometer. A range of MS conditions was tested (e.g., different cone voltages), and the current conditions were those under which spectra could be obtained at sufficient signal-to-noise, where the peak width was not too broad and where the highest oligomeric forms were maintained. Nevertheless, while the prior biochemical methods were unable to ascribe a definitive stoichiometry for a fully assembled RA oligomer, the nanoESI-MS data indicate tobacco RA can form hexamers, the common subunit conformation shared by most AAA+proteins [38, 39].

The influence of $\mathrm{NH}_{4} \mathrm{OAc}$ concentration $(10 \mathrm{mM}-1 \mathrm{M})$ on RA oligomerization was tested (data not shown). At lower $\mathrm{NH}_{4} \mathrm{OAc}$ concentrations, the relative abundance of ions corresponding to higher oligomeric RA forms $(\mathrm{m} / \mathrm{z}$ range 5500-7500) was lower implying self-association of RA monomers may be enhanced at higher ionic strength. Subsequent nanoESI-MS analyses of RA were therefore performed in $500 \mathrm{mM} \mathrm{NH}{ }_{4} \mathrm{OAc}, \mathrm{pH} 7.2$, to enhance the abundance of the higher oligomeric forms. Prior studies have shown that enhancement in RA self-association, and associated catalytic viability, are stimulated by solventexcluding reagents such as polyethylene glycol (PEG) [21]. $\mathrm{K}^{+}$and $\mathrm{Mg}^{2+}$ have also been shown to stimulate RA ATPase activity and subunit association - as judged by increases in intrinsic fluorescence - albeit only requiring saturating ionic concentrations of 22 and $5 \mathrm{mM}$, respectively, which are relevant to physiologic levels in the stroma of chloroplasts [23]. The effect of $\mathrm{Mg}^{2+}$ on RA assembly and activity, however, may arise from an apparent co-factor role since RA appears to stably bind two magnesium ions [40]. It is also possible that high ionic strengths might encourage association of hydrophobic patches of adjacent RA subunits, although this is difficult to ascribe without a molecular structure for a RA holoenzyme.

\section{Interactions of Adenine Nucleotides with Rubisco Activase}

Closer inspection of each ion in Figure $1 \mathrm{~b}$ revealed fine structure. An example is shown in the inset showing the $\mathrm{m} / \mathrm{z}$ region 4700-4900. The ion of low abundance observed at $\mathrm{m} / \mathrm{z}$ $\sim 4754$ is consistent with the $[\mathrm{M}+18 \mathrm{H}]^{18+}$ ion of a dimer of mass $85,534 \mathrm{Da}$. The peak width at half-height of this ion is 8 Th. The broadness of peaks in ESI mass spectra of proteins 


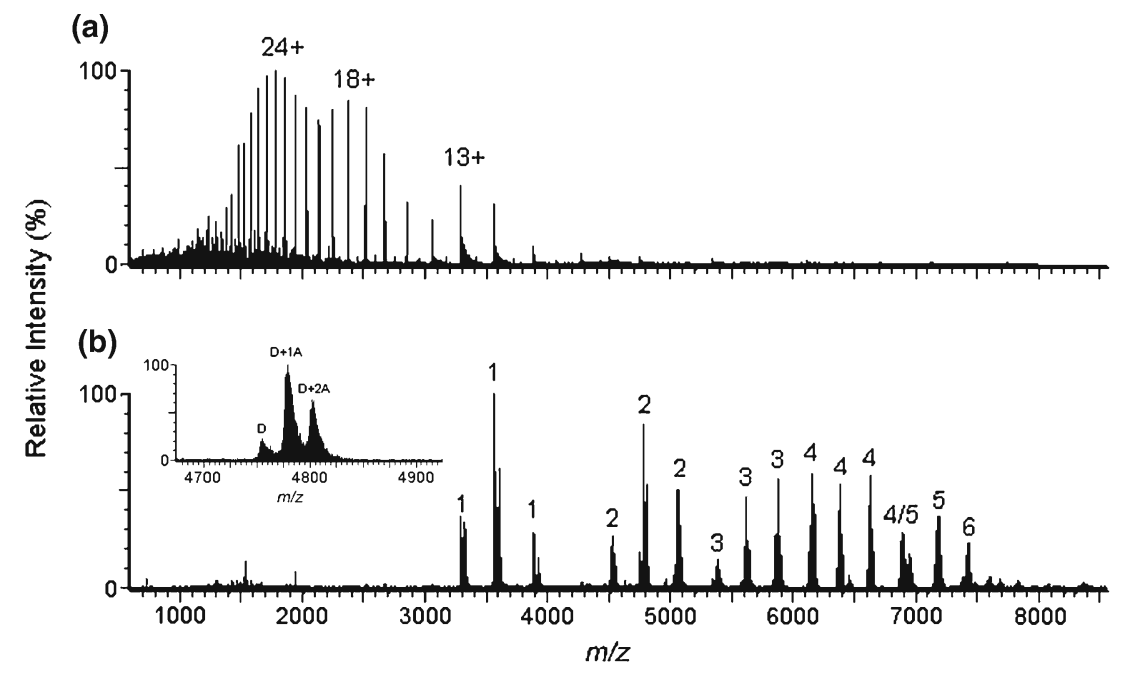

Figure 1. Positive ion nanoESI mass spectra of tobacco RA. (a) In the presence of $10 \mathrm{mM} \mathrm{NH}_{4} \mathrm{OAc}$ in $13 \%$ (vol/vol) acetic acid. The numbers represent the ions directly below them (e.g.; 18+ refers to the $[\mathrm{RA}+18 \mathrm{H}]^{18+}$ ion at $\mathrm{m} / z$ 2377. (b) After the addition of $\mathrm{NH}_{4} \mathrm{OAc}, \mathrm{pH} 7.2$, to $500 \mathrm{mM}$. The numbers above the ions refer to the oligomeric forms that are proposed (e.g.; 6 refers to hexamer). The inset focuses on the $18+$ ion from the RA dimer ( $D$ in the inset); $D+1 A$ and $D+2 A$ refer to the dimer with one and two molecules of ADP bound, respectively

under conditions where they are expected to remain folded arises not only from the presence of isotopes but has also been proposed to arise from the presence of water molecules and solute ions that may be sequestered in the structure [32, 41]. While this provides confidence that the protein detected in the mass spectra is present in its folded form, it also introduces error to the determination of the mass, especially when centered spectra are used to select ions for transformation to a mass scale. For this reason, the values of $\mathrm{m} / \mathrm{z}$ quoted correspond to the value observed to the lower $\mathrm{m} / \mathrm{z}$ side of peaks from ions. There are two ions associated with the dimer. The abundant ion at $\mathrm{m} / \mathrm{z} \sim 4776$ corresponds to a mass of $85,961 \mathrm{Da}$. The ion at $\mathrm{m} / \mathrm{z} 4800$ corresponds to a mass of $86,388 \mathrm{Da}$. The mass difference between each ion corresponds to $427 \mathrm{Da}$ and the mass of ADP is $427.2 \mathrm{Da}$. As RA shows ATPase activity, this suggests that the ATP present during expression in E. coli was hydrolysed to ADP and remained noncovalently bound to RA subunits during their purification, dialysis and acquisition of the nanoESI mass spectra. In this paper, this is referred to as "endogenous ADP." In the case of the dimer, the forms that were present were free dimer (2RA), $2 \mathrm{RA}+1 \mathrm{ADP}$, and $2 \mathrm{RA}+2 \mathrm{ADP}$. This is consistent with one ADP-binding site per monomer. Similar observations were made for all oligomeric forms (up to pentamer). For example, the ions in the $m / z$ region 5250-6000 correspond to trimer (3RA), 3RA+1ADP, 3RA+2ADP and 3RA+3ADP. These observations support the assignment of oligomeric forms in Figure $1 \mathrm{~b}$; however, the ions from hexamer $+n \mathrm{ADP}$ were too low in abundance to assign with certainty. While prior studies using fluorescence quenching with 1-anilinonaphthalene-8sulfonic acid and intrinsic fluorescence have been used to examine differences in the nucleotide binding capacity of modified and unmodified RA [42, 43], our nanoESI-MS measurements uniquely demonstrate the capacity of each RA monomer to bind ADP.

The retention of bound ADP to the purified RA raises concerns as to the suitability of using recombinant RA for nucleotide binding and self-assembly assays of RA. Our nanoESI-MS data indicate that during expression in E. coli the ADP produced from the hydrolysis of ATP remained tightly bound to RA. Based on the abundance of ions from free and ADP-bound RA, $\geq 50 \%$ of the purified RA was present in a complex with ADP. Importantly this may influence the capacity of RA to oligomerize given the inhibitory influence of bound ADP on the ATPase and Rubisco activation activities of RA and the stimulatory effect of ATP on RA self-association [13, 15, 22, 24, 42, 44]. Therefore, in order to be able to examine the effects of added nucleotides on the oligomerization of RA and to compare binding affinities of nucleotides by nanoESI-MS, a method was developed to remove bound ADP from RA.

It was proposed that the high ionic strength would disrupt electrostatic interactions that may be involved in binding the phosphate groups of ADP to the protein. In preliminary experiments, RA was dialysed against $4 \mathrm{M} \mathrm{NH}_{4} \mathrm{OAc}$, $\mathrm{pH} 7.2$, in an attempt to remove bound ADP. Inspection of the nanoESI mass spectrum revealed ions corresponding to RA with bound ADP that were $\sim 0.3$ - to 0.5 -fold the abundance of the corresponding ions from ADP-free RA (not shown). In another attempt, the first dialysis solution was changed to $4 \mathrm{M} \mathrm{NH}_{4} \mathrm{OAc}, \mathrm{pH} 5.5$, and followed by dialysis against $10 \mathrm{mM} \mathrm{NH}{ }_{4} \mathrm{OAc}, \mathrm{pH}$ 7.2. Under these conditions, the nanoESI mass spectra showed that all the ADP was removed (data not shown); however, the ATPase activity of the ADP-free Rubisco activase measured in the enzyme-linked spectrophotometric assay was 200 -fold lower 
than for a control sample that had not been subjected to dialysis at $\mathrm{pH}$ 5.5. Additionally, the $\mathrm{CD}$ spectrum of the Rubisco activase after dialysis at $\mathrm{pH} 5.5$ had lost the features of a control sample characteristic of a predominantly alpha helical structure showing that the protein was unfolded by this treatment (not shown). Dialysis of the purified RA against $40 \mathrm{mM}$ Tris- $\mathrm{HCl}$ buffer, $\mathrm{pH} 7.2,4 \mathrm{M}$ in $\mathrm{NaCl}$ followed by $10 \mathrm{mM} \mathrm{NH} \mathrm{NHAc}_{4} \mathrm{OH} 7.2$, proved more successful. Under these conditions, the ADP was removed; only ions corresponding to ADP-free RA were observed in the nanoESI mass spectrum (not shown). The CD spectrum was indistinguishable from a control sample. ATPase activity (spectrophotometric assay) remained, but was approximately one fifth of that observed for a control sample that had not been subjected to any dialysis steps.

ADP-free RA prepared by dialysis against $4 \mathrm{M} \mathrm{NaCl}$ was used to compare the relative binding affinities of ADP and AMP-PNP. The latter is an analog of ATP in which the oxygen atom linking the $\beta$ - and $\gamma$-phosphate groups in ATP is replaced by $-\mathrm{NH}-$ in AMP-PNP. It is therefore not possible for an ATPase to hydrolyse the $\gamma$-phosphate of AMP-PNP, but the geometry about this group is similar for both nucleotides. The structures of all the nucleotides used in this work are shown in Online Resource 1. ADP-free RA was treated with ADP or AMP-PNP in $500 \mathrm{mM}$ $\mathrm{NH}_{4} \mathrm{OAc}, \mathrm{pH} 7.2$ as this concentration improved the percentage of RA observed in higher oligomeric forms (Figure 1b). The region of the resulting nanoESI mass spectra corresponding to the $[\mathrm{M}+12 \mathrm{H}]^{12+}$ ion of the monomer and associated complexes with nucleotides are shown in Figure $2(\mathrm{~m} / \mathrm{z} 3400-3800)$. In each spectrum, the $[\mathrm{M}+12 \mathrm{H}]^{12+}$ ion of the free RA monomer is present at $\mathrm{m} / \mathrm{z}$ $\sim 3563$. The ion at $\mathrm{m} / z 3599$ is from RA monomer with one ADP molecule bound $[\mathrm{RA}+1 \mathrm{ADP}+12 \mathrm{H}]^{12+}$. Figure $2 \mathrm{a}-\mathrm{c}$ demonstrate how the MS spectra varied as the ADP concentration was increased from $1 \mu \mathrm{M}$ (a) to $10 \mu \mathrm{M}$ (b) to $100 \mu \mathrm{M}$ (c). As the ADP concentration increased, the abundance of the free RA monomer decreased, and the ion from RA+1ADP increased until it was the most abundant ion in the spectrum (Figure 2c). Figure $2 \mathrm{~d}-\mathrm{f}$ show the spectra of RA after reaction with identical AMP-PNP concentrations. The ion at $m / z \sim 3603$ corresponds to one AMP-PNP molecule bound to the RA monomer [RA+ $1 \mathrm{AMP}-\mathrm{PNP}+12 \mathrm{H}]^{12+}$. As the concentration of AMP-PNP was increased, the ion from the free RA monomer remained the most abundant ion, with only a small increase in the abundance of RA+1AMP-PNP. This demonstrates that AMP-PNP, and by inference, ATP, binds much more weakly to tobacco RA than ADP. ATP (magnesium salt) could not be used to compare relative binding affinities to the ADP-free RA since it was rapidly hydrolysed to ADP in the $\mathrm{NH}_{4} \mathrm{OAc}$ solutions used in this work $(10-500 \mathrm{mM}$, $\mathrm{pH}$ 7.2), resulting in mass spectra that showed ions from RA with bound ADP (data not shown). This supports that a substantial proportion of the RA remained folded after the dialysis treatment to remove ADP.
The dependence of the ATPase activity of RA on ADP/ ATP ratio indicate it is inhibited by ADP and that RA is likely inactive in the ADP-bound form [15]. Previous experiments that measured changes in 1-anilinoaphthalene8 -sulfonic acid (ANS) fluorescence upon nucleotide binding showed that at $\mathrm{pH}$ 7.0, ADP had a higher binding affinity for RA than ATP [44]. This is consistent with the mass spectrometry results observed here. This has important implications for the roles of ADP and ATP in RA function. Wang et al. [22] used intrinsic fluorescence to examine structural differences that change the environment around tryptophan residues upon binding of ATP or the nonhydrolysable analogue, adenosine 5'-[ $\gamma$-thio]-triphosphate (ATP $\gamma \mathrm{S}$ ). Inhibition by ADP could be overcome by addition of ATP $\gamma$ S, suggesting competitive binding at the nucleotide binding site of RA [22]. It is interesting to note that when RA was present with $100 \mu \mathrm{M}$ ADP (Figure 2c), a low abundance ion is apparent at $\mathrm{m} / \mathrm{z} 3636$ that corresponds to two ADP molecules bound to the monomer. To investigate this further, an ADP-free sample of RA was treated with $100 \mu \mathrm{M}$ ATP-Mg. The resulting nanoESI mass spectrum showed ions corresponding to free RA monomer, RA+ 1ADP, RA+2ADP, and RA+ADP+ATP (not shown). The presence of bound ADP confirms that the ADP-free RA was able to hydrolyse ATP-Mg in $500 \mathrm{mM} \mathrm{NH} \mathrm{N}_{4} \mathrm{OAc}$, pH 7.2. Further, the detection of RA with two ADP molecules bound or with both ADP and ATP bound suggests that more than one nucleotide binding site may be present on the RA monomer.

The possibility that RA monomer was able to bind ADP and ATP simultaneously, suggesting a possible second nucleotide binding site, was investigated further in a competitive binding experiment. ADP-free RA was treated with: (1) ADP followed by AMP-PNP, (2) AMP-PNP followed by ADP, or (3) simultaneous addition of ADP and AMP-PNP. The resulting spectra were essentially the same independent of the order of addition of the nucleotides (Figure 3). In all three spectra, the ions at $\mathrm{m} / \mathrm{z} 3563,3599$, 3634 , and 3640 correspond to the $[\mathrm{M}+12 \mathrm{H}]^{12+}$ ion of free RA monomer, RA+1ADP, RA $+2 \mathrm{ADP}$, and $\mathrm{RA}+\mathrm{ADP}+$ AMP-PNP, respectively. The relatively high abundance of the ion from RA+1ADP confirms that RA has a higher affinity for ADP than for AMP-PNP. It has been previously reported that addition of excess ATP $\gamma \mathrm{S}$ could overcome inhibition by ADP, however the relative concentrations of nucleotides used were not specified [22]. The MS experiments reported here show that the ADP binds tightly at the first nucleotide binding site. This site does not readily bind AMP-PNP (see Figure $2 \mathrm{f}$ for $100 \mu \mathrm{M}$ AMP-PNP), but binding of ADP appears to stimulate binding of nucleotides at a second binding site. This experiment was repeated several times and the results were reproducible. When $10 \mu \mathrm{M} \mathrm{Mg}(\mathrm{OAc})_{2}$ was included in the reaction mixtures, the results were the same. Spectra of ADP-free RA treated with higher concentrations $(500 \mu \mathrm{M})$ of ADP or AMP-PNP were also examined to determine whether two nucleotides 

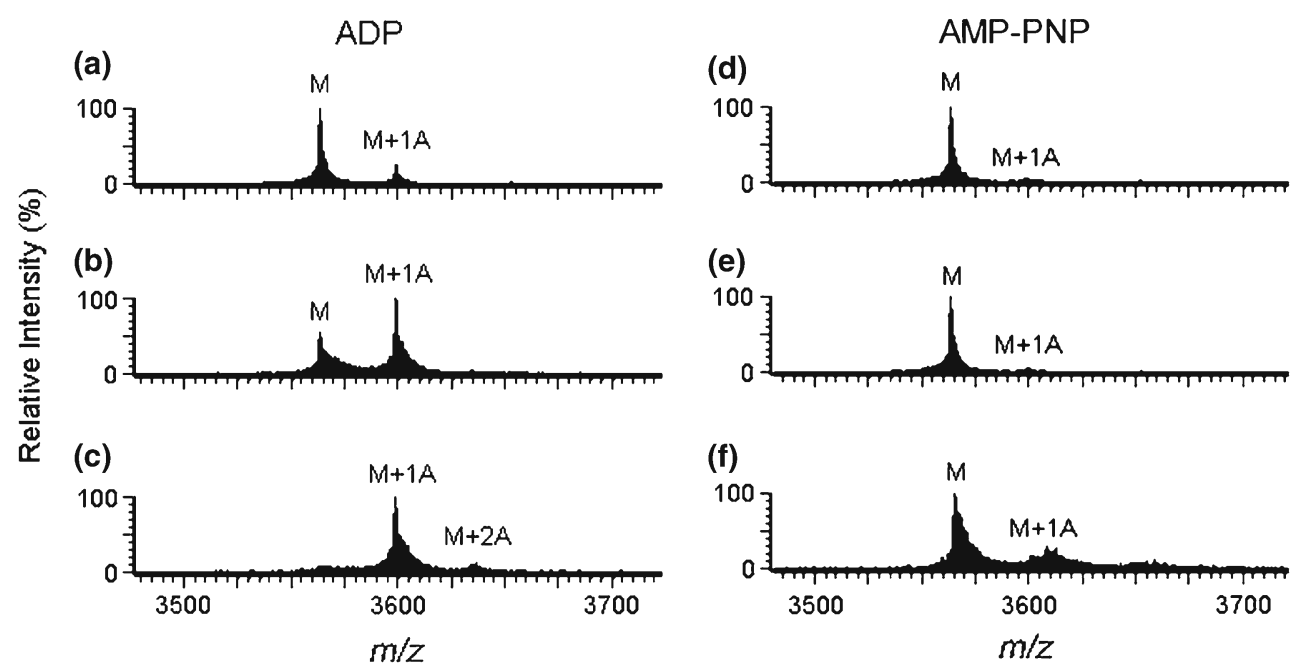

Figure 2. Positive ion nanoESI mass spectra of ADP-free RA treated with ADP or AMP-PNP in $500 \mathrm{mM} \mathrm{NH}_{4} \mathrm{OAc}$, $\mathrm{pH} 7.2$. The $[\mathrm{M}+12 \mathrm{H}]^{12+}$ ion of the monomer is shown; (a) $1 \mu \mathrm{M}$ ADP; (b) $10 \mu \mathrm{M}$ ADP; (c) $100 \mu \mathrm{M}$ ADP; (d) $1 \mu \mathrm{M}$ AMP-PNP; (e) $10 \mu \mathrm{M}$ AMPPNP; (f) $100 \mu \mathrm{M}$ AMP-PNP. M, M+1A, and $M+2 A$ represent ions from the RA monomer, monomer with one ADP molecule bound and monomer with two ADP molecules bound, respectively

were bound to each monomer. Under these conditions the signal-to-noise ratio was significantly reduced making it difficult to distinguish low abundance ions that may have been present (data not shown).

It should be noted that in the $\mathrm{m} / \mathrm{z}$ region $3650-3670$ (Figure 3), there were ions of very low abundance that are consistent with the binding of a third nucleotide. It is likely that these ions result from non-specific interactions and therefore it cannot be ruled out that the ions corresponding to the existence of a second nucleotide binding site also arise from non-specific interactions. The loss of some ATPase activity (spectrophotometric assay) shows that some protein

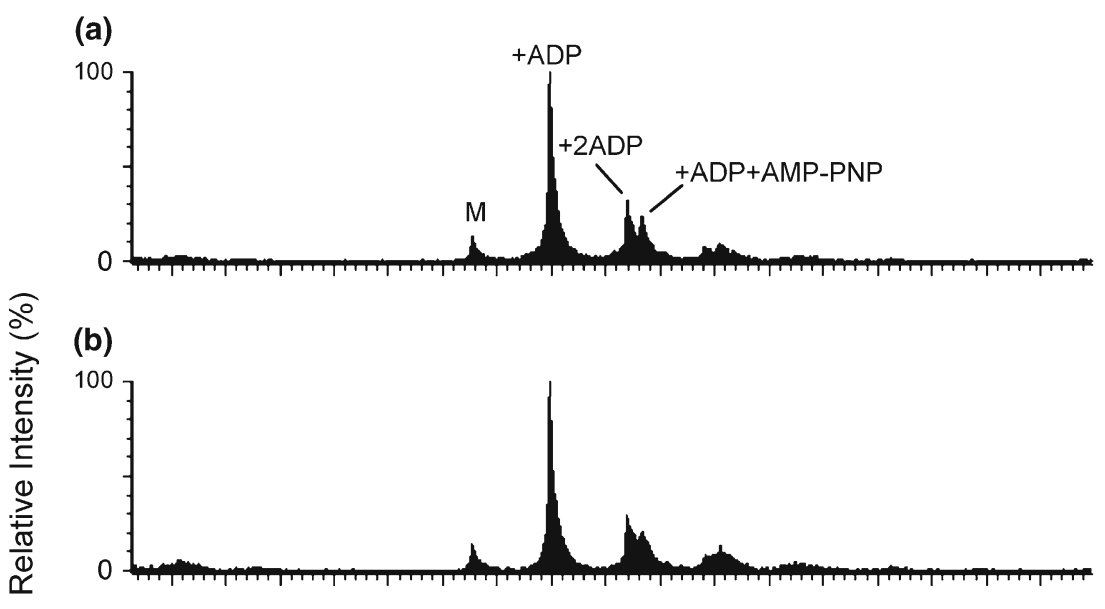

(c)

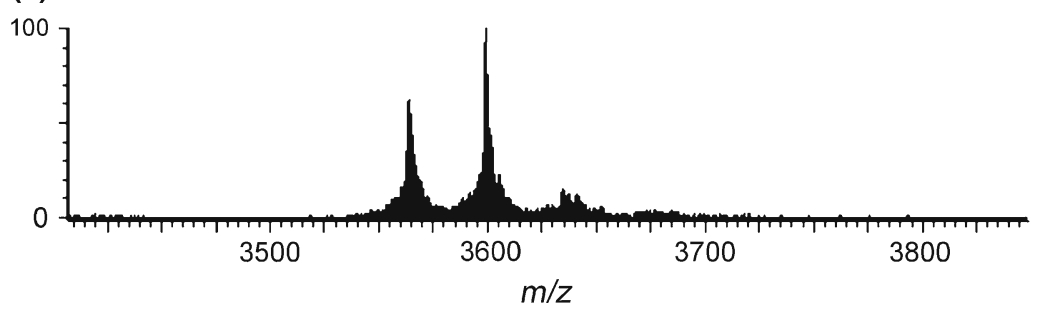

Figure 3. NanoESI mass spectra showing binding of ADP and AMP-PNP to ADP-free RA. The $[R A+12 H]^{12+}$ ion is shown. (a) ADP added first, followed by AMP-PNP. (b) AMP-PNP added first, followed by ADP. (c) ADP and AMP-PNP added simultaneously. The final concentrations of ADP-free RA, ADP, AMP-PNP, and $\mathrm{NH}_{4} \mathrm{OAc}, \mathrm{pH} 7.2$, were $30,100,100$, and $500 \mathrm{mM}$, respectively. $M$ represents RA monomer, +ADP represents RA+1ADP, +2ADP represents RA+2ADP, and+ADP+ AMP-PNP represents RA+ADP+AMP-PNP 
molecules were unfolded by the dialysis treatment to remove endogenous ADP, possibly affecting the ability of RA to bind nucleotides. Nevertheless, when nucleotides were added to RA samples that had not been subjected to the dialysis treatment, the binding of a second nucleotide was also observed in abundances similar to that for RA that had been dialysed to remove endogenous ADP. Collisioninduced dissociation (CID) experiments have been used previously in ESI-MS studies of noncovalent complexes to investigate the stability of the interaction [45, 46]. A study of the interaction between the Tus replication termination protein and the specific DNA-binding sequence, Ter, examined its dissociation under high salt $(800 \mathrm{mM}$ $\mathrm{NH}_{4} \mathrm{OAc}$ ) conditions. These conditions were sufficient to compare dissociation behavior of different Tus mutants. Thus, as the two nucleotide binding sites of RA were observed using $500 \mathrm{mM} \mathrm{NH}_{4} \mathrm{OAc}$ as solvent, it is reasonable to expect that these high salt reaction conditions should minimize weak non-specific binding interactions arising from electrostatic interactions (e.g., the phosphate groups of the nucleotides with arginine or other basic residues).

The possibility for an additional nucleotide binding site on RA supplementary to the ones associated with residues associated with the Walker A motif [24] is also raised by biochemical studies with other enzymes with ATPase activity. The chaperone activity of the homodimeric heat shock protein, Hsp90, is dependent upon the binding and hydrolysis of ATP [47]. An X-ray crystal structure revealed that the ATP-binding site is in the N-terminal domain [47], with recent studies demonstrating a second ATP-binding site in the C-terminal domain that becomes accessible only after the N-terminal site has been occupied [48]. The AAA+ protein, Hsp104, from Saccharomyces cerevisiae also has two nucleotide binding sites [49]. Mutation of an arginine residue in the Sensor 2 region of the second nucleotide binding domain (NBD2) reduced the affinity for both ATP and ADP indicating that NBD2 is involved in nucleotide binding but not in ATP hydrolysis. The finding that the mutation also reduced ATP hydrolysis at NBD1 indicates there is likely communication between the two domains during the ATP hydrolysis cycle. These examples may have implications for the role of the postulated second nucleotide binding site observed in the nanoESI mass spectra for RA.

\section{Interactions of Arginine Mutants of Rubisco Activase with Nucleotides}

Arginine residues serve critical roles in the active sites and subunit interfaces of the ATPase domains of AAA+ proteins. They are proposed to have roles in binding the $\gamma$-phosphate of ATP promoting hydrolysis, in mediating communication among subunits, and in nucleotide sensing [50]. In tobacco RA, the conserved arginine residues R241 and R244 are in the Box VII domains and R294 and R296 are in the Sensor 2 domain [24]. In other AAA+proteins for which detailed molecular structures are available, the highly conserved Arg residues in Box VII closely associate with the $\gamma$-phosphate of ATP from an adjacent subunit when the proteins oligomerize. This facilitates ATP hydrolysis. An arginine in Sensor 2 is thought to mediate a conformational change in the Cterminus in response to the presence of ATP [24 and references therein]. The effects of individually substituting R241, R244, R294, and R296 with alanine on the ATPase activity and oligomerization of tobacco RA have also been examined [24]. Each mutation reduced ATPase activity by more than $70 \%$; however, the mutant RAs appeared to maintain their nucleotide binding affinities as judged by ANS fluorescence.

In the current work, the nucleotide-binding properties of tobacco RA containing single (R241A, R294A, or R296A) and triple (R[241, 244, 294]A) mutations were investigated using nanoESI-MS. The molecular mass of each RA mutant was determined in the presence of acetic acid and the observed monomeric masses of $42672 \mathrm{Da}$ (for the single mutants) and 42,499 $\mathrm{Da}$ (for the triple mutant) were consistent with the expected masses from the amino acid sequences. The mutants were also analysed by nanoESI-MS under conditions previously applied to favour association of subunits $\left(500 \mathrm{mM} \mathrm{NH} \mathrm{N}_{4} \mathrm{OAc}, \mathrm{pH}, 7.2\right)$ and detect endogenous ADP that had remained bound during RA purification (Figure 1b). Figure 4 shows the various spectra for the monomeric $[\mathrm{M}+12 \mathrm{H}]^{12+}$ ion of wild type RA and the R296A and triple RA mutants. The R[241, 244, 294]A triple mutant did not show any ADP binding (a) while the R296A did, albeit with apparently lower affinity (b) than wild type $\mathrm{RA}$ as the RA+1ADP ions were lower in abundance (compare Figure $4 \mathrm{~b}$ with $\mathrm{c}$ ). The amounts of bound ADP for the purified recombinant R241A and R294A mutants were lower than the R296A mutant (data not shown). The approximate percentages of RA monomer present as RA+ $1 \mathrm{ADP}$ were $50 \%, 20 \%, 12 \%, 13 \%$, and 0 for wild type RA, R296A, R241A, R294A, and R[241, 244, 294]A, respectively. When ADP was added to each sample $(100 \mu \mathrm{M})$ additional ADP bound to the wild type, R241A and R294A so that the percentage of RA monomer present as RA+ $1 \mathrm{ADP}$ increased to approximately $60 \%, 55 \%$, and $50 \%$, respectively, but remained unchanged for R296A and R[241, 244, 294]A.

The influence of the mutations on binding of the nonhydrolysable ATP analog ATP $\gamma \mathrm{S}$ was examined by nanoESI-MS (Figure 5). In the mass spectra of wild type and mutant RAs treated with $100 \mu \mathrm{M}$ ATP $\gamma \mathrm{S}$, ions from monomer were observed with endogenously-bound ADP in the relative abundances previously observed (Figure 4) with no evidence that ATP $\gamma \mathrm{S}$ had bound. This is consistent with the relatively low affinity of wild type RA for AMP-PNP (another non-hydrolysable ATP analog) previously observed (Figure 2d-f). Unexpectedly, the spectra in the $\mathrm{m} / \mathrm{z}$ region 6500-8500 for the R294A mutant showed ions corresponding to RA-hexamers with six bound ATP $\gamma \mathrm{S}$ molecules (Figure 5a). This was not observed for the wild type RA (Figure 5b), nor any of the other mutants (data not shown). 

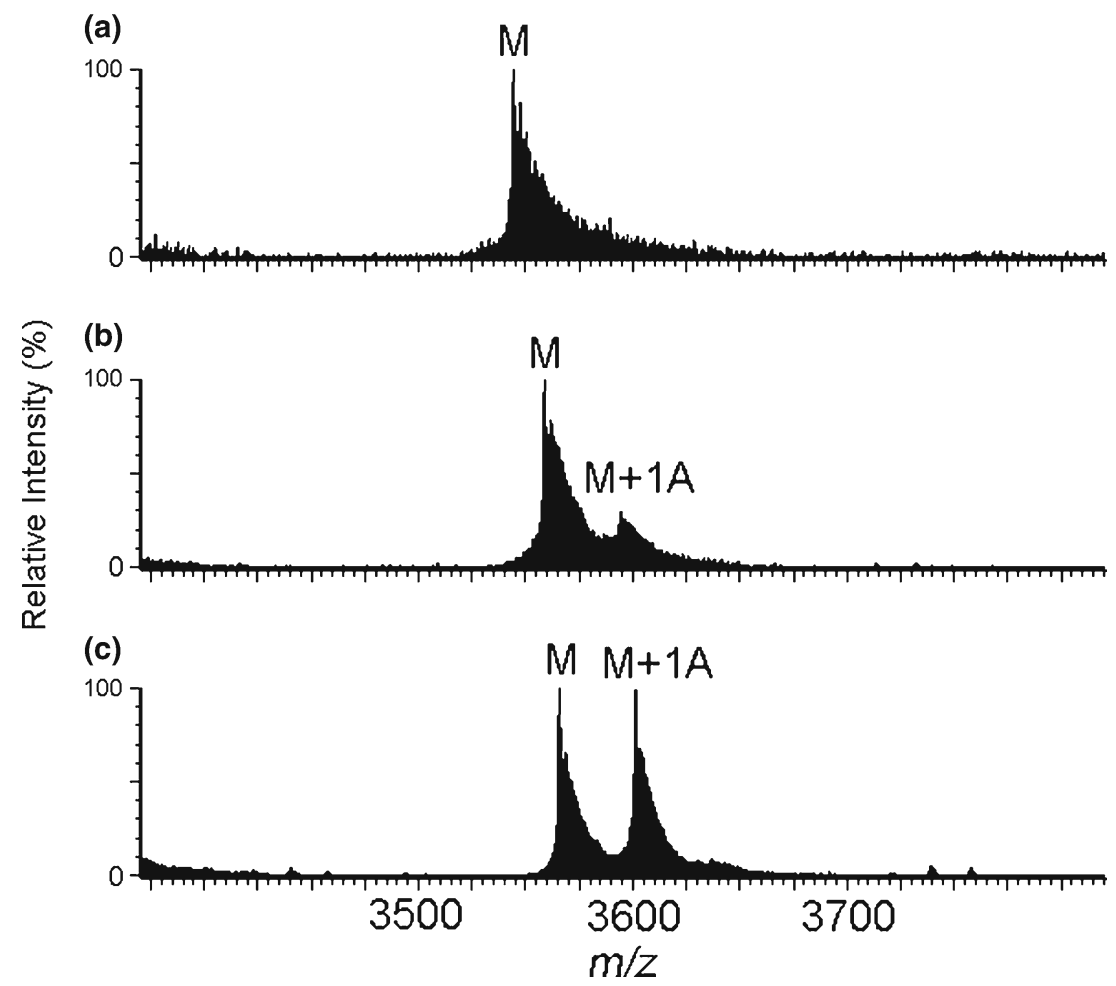

Figure 4. NanoESI mass spectra showing the $m / z$ region for the $[M+12 H]^{12+}$ ion of (a) the triple $R A$ mutant $R[241,244,294] A$, (b) the single RA mutant R296A, and (c) wild type RA. M refers to the monomer (no ADP bound); M+1A refers to monomeric RA with one ADP molecule bound

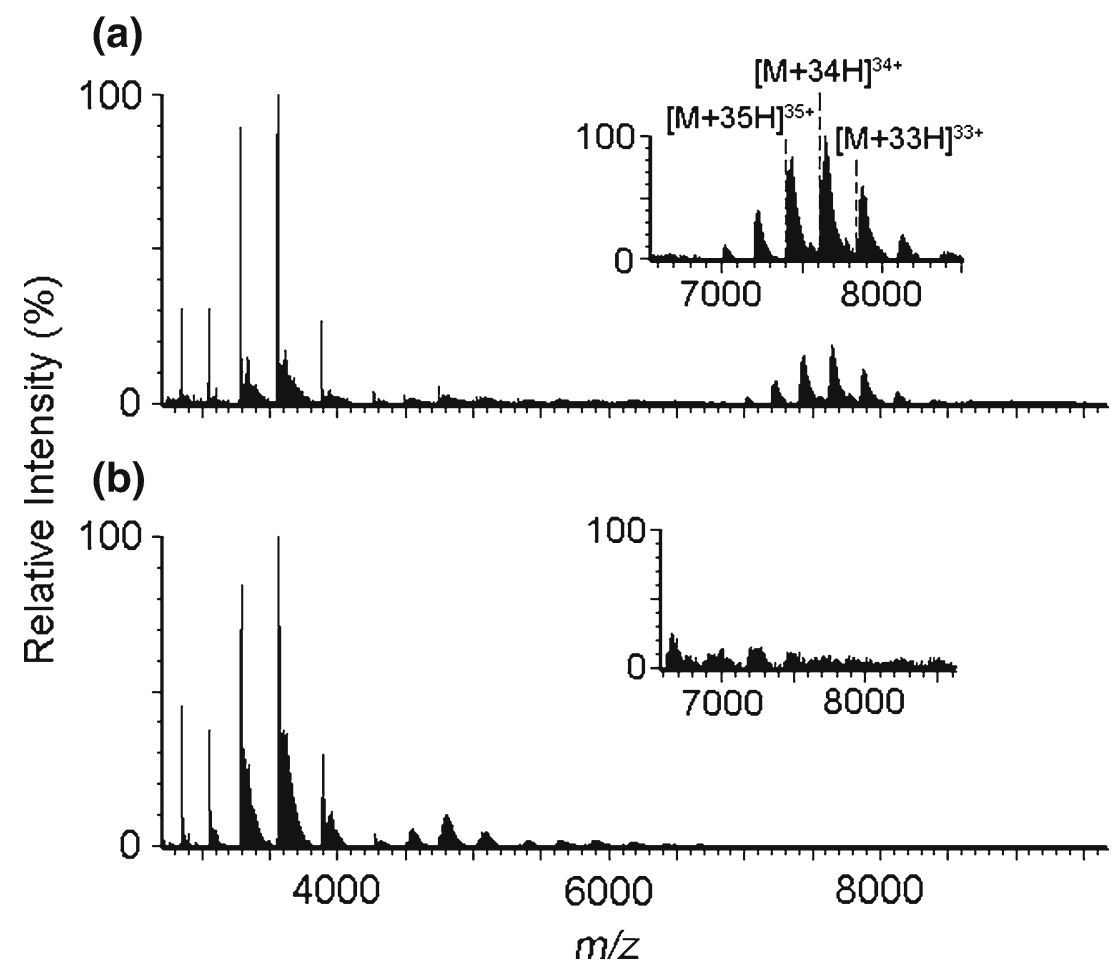

Figure 5. NanoESI mass spectra of (a) the singly mutated R294A, and (b) wild type RA in the presence of $100 \mu M$ ATPYS. The inset shows the $\mathrm{m} / \mathrm{z}$ region $7000-8500$ (hexamer). Note that in the inset in (b), the abundance of the ions in the region presented are enhanced relative to the full spectrum where they are not readily observable. The dotted lines in the insert in (a) show the lower $\mathrm{m} / \mathrm{z}$ side of peaks from the $[\mathrm{M}+35 \mathrm{H}]^{35+},[\mathrm{M}+34 \mathrm{H}]^{34+}$, and $[\mathrm{M}+33 \mathrm{H}]^{33+}$ ions at $\mathrm{m} / \mathrm{z} 7409,7627$, and 7859 , respectively. $\mathrm{M}$ corresponds to R294A RA hexamer+6 ATPyS 
The ions in the $m / z$ region $<4000$ correspond to monomer and approximately $10 \%$ of the R294A signal was present as RA+1ADP. The ions at $\mathrm{m} / \mathrm{z} 4500-5200$ correspond to dimer. Given their low abundance it was not possible to determine whether any ATP $\gamma \mathrm{S}$ was bound. The theoretical mass for a R294A hexamer with six bound ATP $\gamma \mathrm{S}$ molecules is $42,672+6(427)=259,314 \mathrm{Da}$. Therefore, the theoretical $\mathrm{m} / \mathrm{z}$ values for the $[\mathrm{M}+36 \mathrm{H}]^{36+},[\mathrm{M}+35 \mathrm{H}]^{35+}$, $[\mathrm{M}+34 \mathrm{H}]^{34+}$, and $[\mathrm{M}+33 \mathrm{H}]^{33+}$ ions are at $\mathrm{m} / \mathrm{z} 7204,7409$, 7627 , and 7859 , respectively. The $m / z$ values observed in Figure 5 of 7202, 7407, 7625, and 7858 are in agreement with this. This was the first nanoESI mass spectrum observed where the assembly of one oligomeric form of RA was favoured. The preferential assembly into hexamer supports the assertion that like other $\mathrm{AAA}^{+}$ proteins $[39,40]$, this is the optimal subunit stoichiometry of active RA.

Incubation of R294A and the other mutant RAs with $100 \mu \mathrm{M}$ AMP-PNP had no influence on oligomerization. This indicated that like wild-type RA (Figure 2), the arginine mutations had no influence on the low affinity of RA for this non-hydrolysable ATP analog. These experiments were repeated on four occasions and the results were reproducible. $\mathrm{Li}$ et al. [24] noted that relative to wild type RA, the R294A mutant showed a larger increase in intrinsic fluorescence upon addition of ATP suggestive of altered conformational changes to the enzyme. Our nanoESI-MS analyses suggest the heightened conformational change for R294A may correspond to the mutant readily binding ATP (or ATP $\gamma \mathrm{S}$ ) and forming a hexamer.

There are many difficulties in investigating the active form of RA and examining its interaction with Rubisco. This likely arises from its irregular self-assembly into oligomers of different stoichiometries and the likely influence that ionic strength, nucleotide availability, and protein concentration have on its self-association. These factors may contribute to the apparent inability to obtain a crystal structure to date. The determination of conditions here for preparation of a stable R294A RA hexamer may pose a means by which structural information for RA may be obtainable in the near future through crystallography and/or electron microscopy imaging techniques.

\section{NanoESI-MS of Rubisco}

Rubisco is an enzyme of $\sim 530-560 \mathrm{kDa}$ consisting of eight $\sim 52 \mathrm{kDa}$ identical large subunits arranged as a core of four large subunit dimers capped at either end by four $\sim 12-15 \mathrm{kDa}$ small subunits [1,2]. To confirm the mass of the hexadecameric enzyme, the accurate masses of the small and large subunits were determined using nanoESIMS. Rubisco dialysed against $500 \mathrm{mM} \mathrm{NH} \mathrm{N}_{4} \mathrm{OAc}, \mathrm{pH} 7.2$, and treated with $5 \%(\mathrm{vol} / \mathrm{vol})$ acetic acid gave a molecular mass of $14,532 \mathrm{Da}$ for the small subunit of tobacco
Rubisco matching that ascertained previously [51]. No ions for the large subunit were present in the spectrum of the sample prepared in this way, consistent with previous work where mild treatment with acetic acid precipitated the large subunit [52]. A variety of solution conditions were trialled to solubilize the large subunit including addition of formic acid, isopropanol, urea, and different combinations of these. In all spectra only the small subunit was observed. Only when treated with $6 \mathrm{M}$ guanidine hydrochloride followed by extensive dialysis against $0.1 \%$ formic acid was a nanoESI mass spectrum able to show ions from both the small and large subunits corresponding to masses of $14,532 \pm 0.2 \mathrm{Da}$ and $52,504 \pm$ 1.9 Da, respectively. Based on their subunit stoichiometry (and excluding carbamylation and $\mathrm{Mg}^{2+}$ binding to the catalytic sites) the calculated mass for hexadecameric tobacco Rubisco is therefore 536,288 Da.

NanoESI-MS of Rubisco dialysed against $100 \mathrm{mM}$ $\mathrm{NH}_{4} \mathrm{OAc}, \mathrm{pH} 7.2$, was performed to obtain a spectrum of the intact enzyme (Figure 6). The dotted lines show the values of $\mathrm{m} / \mathrm{z}$ calculated for intact Rubisco from the summed mass of its individual subunits $(536,288 \mathrm{Da})$. The values of $m / z$ expected for the $[\mathrm{M}+52 \mathrm{H}]^{52+},[\mathrm{M}+51 \mathrm{H}]^{51+},[\mathrm{M}+50 \mathrm{H}]$ ${ }^{50+}$, and $[\mathrm{M}+49 \mathrm{H}]^{49+}$ ions, are $10314,10516,10726$, and 10945 , respectively. The broadness of the peaks from ions is the result of the contributions from isotopes and possible water or other solvent components sequestered in the structure. This is the first reported ESI mass spectrum of tobacco Rubisco.

A goal of the current research was to prepare and detect a Rubisco-RA complex by nanoESI-MS. The transitory nature of this interaction has thus far frustrated attempts to detect formation of complexes to study their interaction [11]. As RA functions to restore activity to Rubisco in chloroplasts by removing inhibitory sugar phosphates, including substrate RuBP bound to non-carbamylated catalytic sites, catalytically stalled Rubisco-RuBP complexes were prepared. Inactive Rubisco was carefully prepared by extensive dialysis under $\mathrm{CO}_{2}$-free conditions (see Experimental section) to ensure total decarbamylation (i.e., removal of all $\mathrm{Mg}^{2+}$ and the $\mathrm{CO}_{2}$ bound to Lys201) of each catalytic site in Rubisco prior to treating it with RuBP. The nanoESI-MS spectra of the Rubisco before and after incubation with RuBP are shown in Online Resource 2. The difference in mass between the Rubisco (Online Resource 2a) and Rubisco-RuBP (Online Resource $2 b)$ samples is approximately $2480( \pm 20) \mathrm{Da}$. This corresponds to the binding of eight RuBP molecules $(8 \times 310=2480 \mathrm{Da})$ to Rubisco in the inactive RubiscoRuBP complex. This experiment was repeated where the RuBP was substituted for the tight binding inhibitor carboxyarabinitol bisphosphate (CABP, [35]). A mass difference of 2848 Da was measured in the RubiscoCABP sample consistent with the stoichiometric binding of CABP to all eight catalytic sites on Rubisco. This finding attests to the accuracy of the ${ }^{14} \mathrm{C}$-CABP binding 


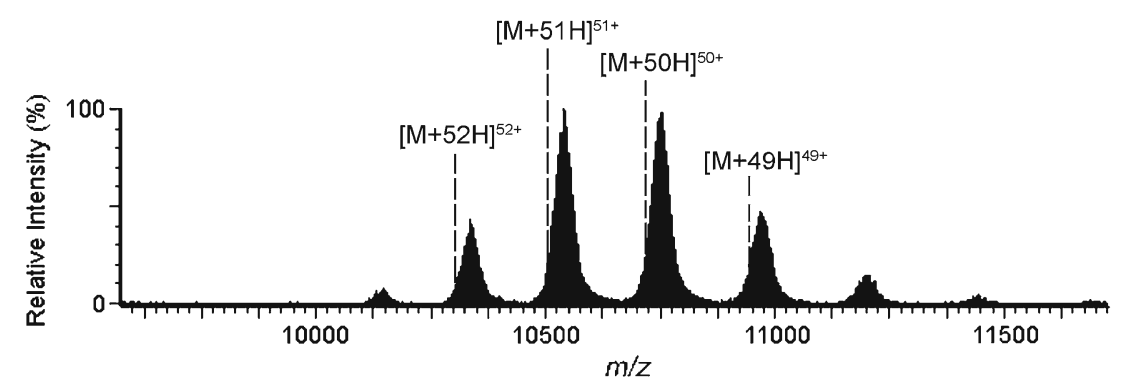

Figure 6. Positive ion nanoESI mass spectrum of hexadecameric tobacco Rubisco dialysed against $100 \mathrm{mM} \mathrm{NH}_{4} \mathrm{OAc}, \mathrm{pH} 7.2$. The dotted lines show the $\mathrm{m} / \mathrm{z}$ values for the $[\mathrm{M}+52 \mathrm{H}]^{52+}$ to $[\mathrm{M}+49 \mathrm{H}]^{49+}$ ions calculated from the combined mass obtained $(536,288 \mathrm{Da})$ from the masses determined experimentally for eight small and eight large subunits. No other ions were observed in the spectrum at values of $\mathrm{m} / \mathrm{z}<10,000$

assay for accurately quantifying Rubisco contents in leaf protein [53] and is contrary to indications CABP may bind sub-stoichiometrically to hexadecameric Rubisco [54].

In attempts to detect RA interacting with Rubisco-RuBP, ADP-free RA was mixed with inactive Rubisco-RuBP complexes with or without addition of ATP-Mg and the nanoESI mass spectra obtained. While no ions corresponding to a Rubisco-RuBP-RA complex were directly observed, the ions for the Rubisco-RuBP complex were shifted to lower $\mathrm{m} / \mathrm{z}$ consistent with RuBP having been removed from Rubisco (i.e., the spectrum for Rubisco was analogous to that shown in Online Resource 2a. The same result was observed when RA with endogenously-bound ADP was used in this experiment. Since this occurred both in the presence or absence of ATP-Mg, it is not clear whether this is indicative of a transient interaction of inactive Rubisco-RuBP with RA resulting in removal of RuBP or whether this is an artifact of the experimental conditions. Future experiments will seek to optimize the experimental conditions used to examine RA activation of Rubisco-RuBP by nanoESI-MS, for example by altering the reaction buffer, nucleotide composition, reaction time, and/or slowing down the activation rate by reducing the RA:Rubisco-RuBP molar ratio. Definition of conditions under which Rubisco-RuBPRA complex(es) can be observed will help to develop hypotheses describing the molecular events involved in Rubisco activation by RA. Notably these studies will be assisted by the availability of the RA arginine mutants since they have diminished Rubisco activation capabilities [24], and in the case of the R294A RA mutant, novel nucleotide binding capabilities (Figure 5).

\section{Conclusions}

These experiments describe the first ESI mass spectra of Rubisco and RA from a higher plant. The spectra show that recombinant RA purified from E. coli can retain bound ADP, which may influence the interpretation of nucleotide binding analyses on RA function that use comparable sources of recombinant enzyme. The tight binding of ADP to RA necessitated dialysis against high ionic concentrations to evince its removal and was further evidenced by the persistence of bound ADP even upon addition of AMP-PNP up to $500 \mu \mathrm{M}$. A second binding site was uniquely observed in RA samples when nucleotides were added, both in RA samples that had or had not been subjected to the dialysis procedure to remove endogenous ADP. It has been proposed that nucleotides can interact at subunit interfaces of some $\mathrm{AAA}+$ proteins, in a location distinct from the ATP-binding domain of the active site. Since RA is a member of the AAA+ proteins, a second nucleotide binding site in RA may be biologically relevant rather than the result of nonspecific binding interactions occurring in the ionization source of the mass spectrometer. This proposal remains to be verified or refuted by the determination of an X-ray structure. Current efforts are being directed towards optimizing the experimental conditions used to prepare RA and examine its nucleotide interactions using continuous dialysis/ultrafiltration of RA against/in the presence of AMP-PNP or ATP $\gamma \mathrm{S}$.

The formation of a stable hexamer of R294A RA in the presence of ATP $\gamma \mathrm{S}$ was an unexpected result. While nanoESI-MS spectra of wild-type RA indicated hexamer formation (Figure 1b), the apparently stable R294A-ATP $\gamma \mathrm{S}$ hexamers were not formed for any of the other conserved arginine mutants or wild type RA and did not form for any of the proteins, including R294A, in the presence of AMPPNP. This result implies that ATP $\gamma$ S can behave differently to AMP-PNP and thus presents a cautionary note for interpretation of nucleotide binding experiments aimed at investigating the mechanism of action (the sequence of events involving ATP-binding hydrolysis, conformational changes of the chaperone and its target) of RA or any nucleotide-binding protein. It remains to be tested whether the ability to prepare the stable R294A-ATP $\gamma \mathrm{S}$ hexamer may be useful in preparing a Rubisco-RuBP-RA complex or provide a means to obtain structural data for RA-hexamers by crystallography or electron microscopy methods. For example, it may be possible to treat catalytically inhibited Rubisco-RuBP complexes with hexameric R294A RA$\mathrm{ATP} \gamma \mathrm{S}$ and detect a complex that is stable to mass spectrometry. These future experiments may also benefit from more detailed nucleotide binding studies of all the arginine RA mutants. 
In plants, the catalytic inadequacies of Rubisco and the limiting thermostability of RA are features that often pose rate limiting steps in photosynthesis and thus are key targets for optimization to improve crop productivity $[1,2,7,20]$. Improving our understanding of RA structure, nucleotide binding requirements for activity, and interaction with Rubisco are therefore paramount towards being able to engineer benefits to both enzymes in crops. Towards this goal, here we have demonstrated that nanoESI-MS provides a useful new tool for providing novel structure-function information on Rubisco and RA.

\section{Acknowledgments}

The authors acknowledge support for this research by SMW by ARC grant FT0991407. They thank Sara Milward, Heather Kane, and Robert Sharwood for their research assistance. J.L.B. acknowledges the University of Wollongong for support and the ARC for the mass spectrometers used in this work. M.J.B. acknowledges receipt of an Australian Postgraduate Award.

\section{References}

1. Whitney, S.M., Houtz, R.L., Alonso, H.: Advancing our Understanding and Capacity to Engineer Nature's $\mathrm{CO}_{2}$-Sequestering Enzyme. Rubisco. Plant Physiol. 155, 27-35 (2011)

2. Andersson, I., Backlund, A.: Structure and Function of Rubisco. Plant Physiol. Biochem. 46, 275-291 (2008)

3. Bassham, J., Benson, A., Calvin, M.: The Path of Carbon in Photosynthesis. J. Biol. Chem. 185, 781-787 (1950)

4. Cleland, W.W., Andrews, T.J., Gutteridge, S., Hartman, F.C., Lorimer, G.H.: Mechanism of Rubisco- the Carbamate as a General Base. Chem. Rev. 98, 549-561 (1998)

5. Spreitzer, R., Salvucci, M.: Rubisco, Structure, Regulatory Interactions, and Possibilities for a Better Enzyme. Annu. Rev. Plant Biol. 53, 449475 (2002)

6. Ellis, R.: The Most Abundant Protein in the World. Trends in Biochem. Sci. 4, 241-244 (1979)

7. Raines, C.A.: Increasing Photosynthetic Carbon Assimilation in $\mathrm{C}_{3}$ Plants to Improve Crop Yield, Current and Future Strategies. Plant Physiol. 155, 36-42 (2011)

8. Portis, A.J.: The Rubisco Activase-Rubisco System, An ATPaseDependent Association that Regulates Photosynthesis. In: McManus, M., Laing, W., Allan, A. (eds.) Protein-Protein Interactions in Plant Biology, pp. 30-52. Sheffield Academic Press, Sheffield (2002)

9. Salvucci, M., Ogren, W.: The Mechanism of Rubisco Activase, Insights from Studies of the Properties and Structure of the Enzyme. Photosynth. Res. 47, 1-11 (1996)

10. Jordan, D., Chollet, R.: Inhibition of Ribulose Bisphosphate Carboxylase by Substrate Ribulose-1,5-Bisphosphate. J. Biol. Chem. 258, 13752-13758 (1983)

11. Portis Jr., A.R., Li, C., Wang, D., Salvucci, M.E.: Regulation of Rubisco Activase and its Interaction with Rubisco. J. Exp. Bot. 59, 1597-1604 (2008)

12. Wang, Z., Portis, A.J.: Dissociation of Ribulose-1,5-Bisphosphate Bound to Ribulose-1,5-Bisphosphate Carboxylase/Oxygenase and Its Enhancement by Ribulose-1,5-Bisphosphate Carboxylase/Oxygenase ActivaseMediated Hydrolysis of ATP. Plant Physiol. 99, 1348-1353 (1992)

13. Portis, A.J.: Rubisco Activase - Rubisco's Catalytic Chaperone. Photosynth. Res. 75, 11-27 (2003)

14. Robinson, S., Streusand, V., Chatfield, J., Portis, A.J.: Purification and Assay of Rubisco Activase from Leaves. Plant Physiol. 88, 1008-1014 (1988)

15. Robinson, S., Portis, A.J.: Adenosine Triphosphate Hydrolysis by Purified Rubisco Activase. Arch. Biochem. Biophys. 268, 93-99 (1989)
16. Neuwald, A., Aravind, J., Spouge, J., Koonin, E.: AAA+, A Class of Chaperone-Like ATPases Associated with the Assembly, Operation, and Disassembly of Protein Complexes. Genome Res. 9, 27-43 (1999)

17. Ogura, T., Wilkinson, A.: AAA+Superfamily ATPases. Common Structure-Diverse Function. Genes Cells 6, 575-597 (2001)

18. Hanson, P., Whiteheart, S.: AAA+Proteins, Have Engine. Will Work. Nat. Rev. Mol. Cell Biol. 6, 519-529 (2005)

19. Zhang, N., Portis, A.R.: Mechanism of Light Regulation of Rubisco, a Specific Role for the Larger Rubisco Activase Isoform Involving Reductive Activation by Thioredoxin-f. Proc. Natl. Acad. Sci. U.S.A. 96, 9438-9443 (1999)

20. Kurek, I., Chang, T.K., Bertain, S.M., Madrigal, A., Liu, L., Lassner, M.W., Zhu, G.H.: Enhanced Thermostability of Arabidopsis Rubisco Activase Improves Photosynthesis and Growth Rates under Moderate Heat Stress. Plant Cell 19, 3230-3241 (2007)

21. Salvucci, M.: Subunit Interactions of Rubisco Activase, Polyethylene Glycol Promotes Self-Association, Stimulates ATPase and Activation Activities, and Enhances Interactions with Rubisco. Arch. Biochem. Biophys. 298, 688-696 (1992)

22. Wang, Z., Ramage, R., Portis, A.J.: $\mathrm{Mg}^{2+}$ and ATP or Adenosine $5^{\prime}-[\gamma-$ thio]-Triphosphate (ATP $\gamma \mathrm{S})$ Enhances Intrinsic Fluorescence and Induces Aggregation, which Increases the Activity of Spinach Rubisco Activase. Biochim. Biophys. Acta 1202, 47-55 (1993)

23. Lilley, R., Portis, A.J.: ATP Hydrolysis Activity and Polymerization State of Ribulose-1,5-Bisphosphate Carboxylase Oxygenase Activase. Plant Physiol. 114, 605-613 (1997)

24. Li, C., Wang, D., Portis, A.J.: Identification of Critical Arginine Residues in the Functioning of Rubisco Activase. Arch. Biochem. Biophys. 450, 176-182 (2006)

25. Portis, A.J.: The Regulation of Rubisco by Rubisco Activase. J. Exp. Bot. 46, 1285-1291 (1995)

26. Larson, E., O'Brien, C., Zhu, G., Spreitzer, R., Portis, A.J.: Specificity for Activase is Changed by a Pro- 89 to Arg Substitution in the Large Subunit of Ribulose-1,5-Bisphosphate Carboxylase/Oxygenase. J. Biol. Chem. 272, 17033-17037 (1997)

27. Yokota, A., Tsujimoto, N.: Characterization of Ribulose-1,5-Bisphosphate Carboxylase/Oxygenase Carrying Ribulose 1,5-Bisphosphate at its Regulatory Sites and the Mechanism of Interaction of this Form of the Enzyme with Ribulose-1,5-Bisphosphate-Carboxylase/Oxygenase Activase. Eur. J. Biochem. 204, 901-909 (1992)

28. de Jiménez, S.: E.; Medrano, L.; Martínez-Barajas, E. Rubisco Activase, a Possible New Member of the Molecular Chaperone Family. Biochemistry 34, 2826-2831 (1995)

29. Esau, B., Snyder, G., Portis, A.J.: Activation of Ribulose-1,5-Bisphosphate Carboxylase/Oxygenase (Rubisco) with Chimeric Activase Proteins. Photosynth. Res. 58, 175-181 (1998)

30. Andrews, T., Hudson, G., Mate, C., von Caemmerer, S., Evans, J., Arvidsson, Y.: Rubisco, the Consequences of Altering its Expression and Activation in Transgenic Plants. J. Exp. Bot. 46, 1293-1300 (1995)

31. Benesch, J.L.P., Robinson, C.V.: Dehydrated but Unharmed. Nature 462, 576-577 (2009)

32. Watt, S.J., Urathamakul, T., Schaeffer, P., Williams, N., Sheil, M. M., Dixon, N.E., Beck, J.L.: Multiple Oligomeric Forms of Escherichia coli DnaB Helicase Revealed by Electrospray Ionization Mass Spectrometry. Rapid Commun. Mass Spectrom. 21, 132-140 (2007)

33. Batchelor, J.D., Sterling, H.J., Hong, E., Williams, E.R., Wemmer, D. E.: Receiver Domains Control the Active State Stoichiometry of Aquifex aeolicus $\sigma(54)$ Activator NtrC4, as Revealed by ESI-MS. J. Mol. Biol. 393, 634-643 (2009)

34. Kane, H.J., Wilkin, J.M., Portis, A.R., Andrews, T.J.: Potent Inhibition of Ribulose-Bisphosphate Carboxylase by an Oxidized Impurity in Ribulose-1,5-Bisphosphate. Plant Physiol. 117, 10591069 (1998)

35. Pierce, J., Tolbert, N.E., Barker, R.: Interaction of Ribulosebisphosphate Carboxylase/Oxygenase with Transition-State Analogues. Biochemistry 19, 934-942 (1980)

36. Servaites, J.: Crystalline Ribulose Bisphosphate Carboxylase/Oxygenase of High Integrity and Catalytic activity from Nicotiana tabacum. Arch. Biochem. Biophys. 238, 154-160 (1985)

37. Baker, R.T., Catanzariti, A.M., Karunasekara, Y., Soboleva, T.A., Sharwood, R., Whitney, S., Board, P.G.: Using Deubiquitylating Enzymes as Research Tools. Methods Enzymol. 398, 540-554 (2005) 
38. White, S.R., Lauring, B.: AAA+ATPases, Achieving Diversity of Function with Conserved Machinery. Traffic 8, 1657-67 (2007)

39. Snider, J., Thibault, G., Houry, W.A.: The AAA+Superfamily of Functionally Diverse Proteins. Genome Biol. 9, 216 (2008)

40. Frasch, W., Spano, M., LoBotto, R.: $\mathrm{VO}^{2+}$ as a Probe of Metal Binding Sites in Rubisco Activase. In: Mathis, P. (ed.) Photosynthesis, from Light to Biosphere, pp. 257-260. Kluwer Academic Publishers, Dordrecht (1995)

41. Rostom, A.A., Fucini, P., Benjamin, D.R., Juenemann, R., Nierhaus, K. H., Hartl, F.H., Dobson, C.M., Robinson, C.V.: Detection and Selective Dissociation of Intact Ribosomes in a Mass Spectrometer. Proc. Natl. Acad. Sci. U.S.A. 97, 5185-5190 (2000)

42. Wang, D., Portis, A.J.: Increased Sensitivity of Oxidized Large Isoform of Ribulose-1,5-Bisphosphate Carboxylase/Oxygenase (Rubisco) Activase to ADP Inhibition is Due to an Interaction Between Its Carboxyl Extension and Nucleotide-Binding Pocket. J. Biol. Chem. 281, 2524125249 (2006)

43. Shen, J., Orozco Jr., E., Ogren, W.: Expression of the Two Isoforms of Spinach Ribulose 1,5-Bisphosphate Carboxylase Activase and Essentiality of the Conserved Lysine in the Consensus Nucleotide-Binding Domain. J. Biol. Chem. 266, 8963-8968 (1991)

44. Wang, Z., Portis, A.J.: A Fluorometric Study with 1-Anilinoaphthalene8-Sulfonic Acid (ANS) of the Interactions of ATP and ADP with Rubisco Activase. Biochim. Biophys. Acta 1079, 263-267 (1991)

45. Schwartz, B., Bruce, J., Anderson, G.S.H., Rockwood, A., Smith, R.: Dissociation of Tetrameric Ions of Noncovalent Streptavidin Complexes Formed by Electrospray Ionization. J. Am. Soc. Mass Spectrom. 6, 459465 (1995)

46. Kapur, A., Beck, J., Brown, S., Dixon, N., Sheil, M.: Use of Electrospray Ionization Mass Spectrometry to Study Binding Interactions Between a Replication Terminator Protein and DNA. Protein Sci. 11, 147-157 (2002)
47. Soti, C., Vermes, A., Haystead, T., Csermely, P.: Comparative Analysis of the ATP-Binding Sites of Hsp90 by Nucleotide Affinity Cleavage, a Distinct Nucleotide Specificity of the C-Terminal ATP-Binding Site. Eur. J. Biochem. 270, 2421-2428 (2003)

48. Soti, C., Racz, A., Csermely, P.A.: Nucleotide-Dependent Molecular Switch Controls ATP Binding at the C-Terminal Domain of Hsp90. NTerminal Nucleotide Binding Unmasks a C-Terminal Binding Pocket. $J$. Biol. Chem. 277, 7066-7075 (2002)

49. Hattendorf, D., Lindquist, S.: Analysis of the AAA Sensor-2 Motif in the C-Terminal ATPase Domain of Hsp104 with a Site-Specific Fluorescent Probe of Nucleotide Binding. Proc. Natl. Acad. Sci. U.S. A. 99, 2732-2737 (2002)

50. Ogura, T., Whiteheart, S.W., Wilkinson, A.J.: Conserved Arginine Residues Implicated in ATP Hydrolysis, Nucleotide-Sensing, and InterSubunit Interactions in AAA and AAA+ATPases. J. Struct. Biol. 146, $106-112$ (2004)

51. Whitney, S.M., Andrews, T.J.: The Gene for the Ribulose-1,5Bisphosphate Carboxylase/Oxygenase (Rubisco) Small Subunit Relocated to the Plastid Genome of Tobacco Directs the Synthesis of Small Subunits that Assemble into Rubisco. Plant Cell 13, 193-205 (2001)

52. Andrews, T., Lorimer, G.: Catalytic Properties of a Hybrid Between Cyanobacterial Large Subunits and Higher Plant Small Subunits of Ribulose Bisphosphate Carboxylase-Oxygenase. J. Biol. Chem. 260, 4632-4636 (1985)

53. Ruuska, S., Andrews, T.J., Badger, M.R., Hudson, G.S., Laisk, A., Price, G.D., von Caemmerer, S.: The Interplay Between Limiting Processes in C-3 Photosynthesis Studied by Rapid-Response Gas Exchange using Transgenic Tobacco Impaired in Photosynthesis. Aust. J. Plant Physiol. 25, 859-870 (1998)

54. Butz, N.D., Sharkey, T.D.: Activity Ratios of Ribulose-1,5-Bisphosphate Carboxylase Accurately Reflect Carbamylation Ratios. Plant Physiol. 89, 735-739 (1989) 